NASA DI 70654

\title{
THERMAL STRUCTURE OF THE MARTIAN ATMOSPHERE DURING THE DISSIPATION OF THE DUST STORM OF 1971
}

(NASA-TH-X-70654) THERHAL STRUCTURE OF

\section{BARNEY J. CONRATH}

APRIL 1974

GSFC GODDARD SPACE FLIGHT CENTER

GREENBELT, MARYLAND 
$\mathrm{X}-622-74-111$

PREPRINT

THERMAL STRUCTURE OF THE MARTIAN ATMOSPHERE DURING THE DISSIPATION

OF THE DUST STORM OF 1971

Barney J. Conrath

April 1974

Goddard Space Flight Center

Greenbelt, Md. 20711 


\title{
THERMAL STRUCTURE OF THE MARTIAN \\ ATMOSPHERE DURING THE DISSIPATION \\ OF THE DUST STORM OF 1971
}

\begin{abstract}
The secular variation of the thermal structure of the Martian atmosphere during the dissipation phase of the 1971 dust storm is examined, using temperatures obtained by the infrared spectroscopy investigation on Mariner 9. For the latitude range $-20^{\circ}$ to $-30^{\circ}$, the mean temperature at the $2 \mathrm{mb}$ level is found to decrease from approximately $220 \mathrm{~K}$ in mid-December 1971 to about $190 \mathrm{~K}$ by June 1972 while for the $0.3 \mathrm{mb}$ level a decrease from $203 \mathrm{~K}$ to $160 \mathrm{~K}$ is observed. Over the same period, the amplitude of the diurnal temperature wave also decreased. Assuming a simplified radiative heating model, the dust optical depth is found to decrease approximately exponentially with an e-folding time of about 60 days at both the 0.3 and $2 \mathrm{mb}$ levels. Stokes-Cunningham settling alone cannot account for this behavior. Sedimentation models which include both gravitational settling and vertical mixing are developed in an effort to explain the time evolution of the dust. Within the framework of a model which assumes an effective vertical diffusivity $\mathrm{K}$ independent of height, a mean dust particle diameter of $\sim 2 \mu \mathrm{m}$ is inferred. To provide the necessary vertical mixing, $\mathrm{K} \gtrsim 10^{7} \mathrm{~cm}^{2}$ sec ${ }^{-1}$ is required in the lower atmosphere.
\end{abstract}

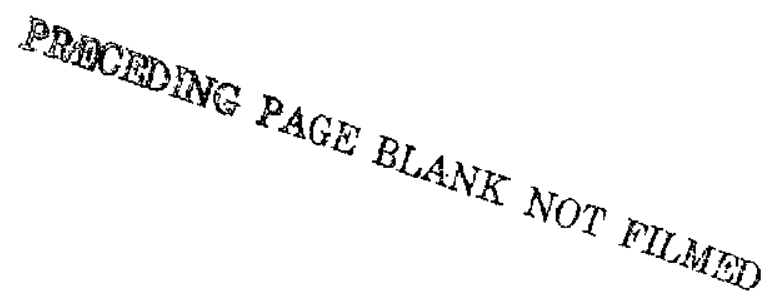




\section{THERMAL STRUCTURE OF THE MARTIAN \\ ATMOSPHERE DURING THE DISSIPATION \\ OF THE DUST STORM OF 1971}

\section{Introduction}

The Mariner 9 spacecraft achieved orbit about Mars 14 Nov. 1971 during a planet-wide dust storm of an extraordinary magnitude. In television pictures returned by the spacecraft during the first few weeks of the mission, the surface of the planet was found to be almost totally obscured. During the later part of December, a clearing trend began with surface features becoming increasingly well defined. By mid-January, surface features were visible almost everywhere, even at large viewing angles (Leovy, et al., 1972).

Data obtained from the infrared spectroscopy investigation on Mariner 9 have been used to obtain atmospheric temperature profiles during the dust storm and its subsequent decay (Hanel, et al., 1972a; 1972b; Conrath, et al., 1973). Data acquired during the storm revealed that the atmosphere was in a highly perturbed thermal state compared to that expected for a clear atmosphere on the basis of theoretical models (Gierasch and Goody, 1969). This suggested that absorption of solar energy by the dust was strongly affecting the atmospheric energy balance, (Gierasch and Goody, 1972). A diurnal temperature wave with a peak-to-peak amplitude of $\sim 30 \mathrm{~K}$ was observed at all atmospheric levels up to at least $40 \mathrm{~km}$, and mean temperatures were substantially higher than those predicted for a clear atmosphere. These observations have been used to deduce the properties of the strong thermally driven atmospheric tides which existed during the storm (Leovy, et al., 1973; Pirraglia and Conrath, 1974)。 
In the present paper, the secular behavior of the atmospheric temperature duriug the clearing poriod following the storm is examined. The data are interpreted in terms of a simplified atmospheric heating model from which a time dependent dust optical depth is deduced. In an effort to explain the behayior of the optical depth as a function of time, a model describing the dynamics of the dust settling process is invoked. This model contains both gravitational settling and vertical mixing of the dust particles and is used to estimate particle size and effective vertical diffusivity.

\section{Data Analysis}

Temperature profiles were calculated using measurements from selected spectral intervals within the $667 \mathrm{~cm}^{-1} \mathrm{CO}_{2}$ absorption band. The techniques employed are similar to those developed for use in the terrestrial meteorological satellite program (see for example Conrath and Revah, 1972). Temperatures were obtained from the surface to $\sim 0.1 \mathrm{mb}$ with a vertical resolution on the order of half of a pressure scale height.

The temperature data were organized according to latitude and local time. In order to define the secular trend, the data prior to orbit 220 (the first 110 days of the mission) were averaged over intervals of approximately six days. Examples of these averages are shown in Figures 1 and 2 for the $0.3 \mathrm{mb}$ and $2 \mathrm{mb}$ atmospheric levels respectively in the latitude interval $-20^{\circ}$ to $-30^{\circ}$. Following orbit 220 , a much smaller quantity of data was acquired, necessitating the use of individual data points rather than averages. Nevertheless, the continuing trend of the data appears to be well defined. 
Examination of Figures 1 and 2 reveals that the diurnal mean temperature at both levels decreased monotonically approaching $\sim 160 \mathrm{~K}$ at $0.3 \mathrm{mb}$ and $\sim 90 \mathrm{~K}$ at $2 \mathrm{mb}$. Comparison of the behavior of data taken in early morning with that in late afternoon permits an estimate of the amplitude of the diurnal wave which is also found to decrease with time. This suggests that the presence of dust is responsible for enhanced atmospheric heating. To relate the temporal behavior of the atmospheric thermal structure directly to the behavior of the dust, it is necessary to first develop an atmospheric heating model.

\section{Radiative Heating Model}

A highly simplified radiative heating model will now be considered. It is assumed that the heat equation can be written

$$
\frac{\partial T}{\partial t}=-\frac{\left(T-T_{e}\right)}{t_{r}}+\frac{Q_{D}}{C_{p} \rho}
$$

where $T$ is atmospheric temperature which is a function of height $z$ and time $t$, $T_{e}$ is the radiative-convective equilibrium temperature which the atmosphere would assume in the absence of dust, $\rho$ is atmospheric density, $C_{p}$ is specific heat at constant pressure, $t_{t}$ is the radiative-convective time constant of the - atmosphere and $Q_{D}$ is atmospheric heating due to the dust. It is assumed that the perturbed thermal state of the atmosphere is produced by direct deposition of solar energy into the atmosphere due to the presence of the dust. The volumetric heating is approximated by

$$
Q_{D}=-\alpha \frac{d F}{d z}
$$


where $F$ is the solar flux reaching level $z$ which can be expressed in the form

$$
\mathbf{F}= \begin{cases}\mu \mathrm{S} e^{-\tau / \mu} ; & 0 \leq \mu \leq 1 \\ 0 \quad & ;-1 \leq \mu \leq 0 .\end{cases}
$$

$\mathrm{S}$ is the solar flux at the top of the atmosphere, $\tau$ is the extinction optical depth and $\mu$ is the cosine of the solar zenith angle. It is assumed that a fraction $a$ of the solar energy removed from the beam goes into heating the atmosphere and solar energy reflected from the surface has been neglected. A similar heating model has been used previously by Leovy, et al. (1972).

With the above assumptions, the heating rate per gram of atmosphere required in (1) can be written

$$
\frac{\mathrm{Q}_{\mathrm{D}}}{\rho \mathrm{C}_{\mathrm{p}}}= \begin{cases}\mathrm{G}(\mathrm{z}, \mathrm{t}) \mathrm{e}^{-\tau(z, t) / \mu(\mathrm{t})} ; & 0 \leq \mu \leq 1 \\ 0 & ;-1 \leq \mu \leq 0\end{cases}
$$

with the definition

$$
G(z, t)=\frac{\sigma \alpha S(t)}{C_{p}} q(z, t)
$$

where $q(z, t)$ is the specific concentration of the dust number of dust particles per gram of atmosphere) and $\sigma$ is the extinction cross section for the dust. Use has been made of the relationship between optical depth and specific concentration which can be written

$$
\tau(z, t)=\int_{1}^{\infty} \operatorname{cg} \rho \mathrm{d} z
$$


The time dependent parameters of the model vary on two distinct time scales: The cosine of the solar zenith angle at colatitude $\theta$ and time $\mathbf{t}$ is given by

$$
\mu(t)=b(t) \cos \Omega t+c(t)
$$

where $\Omega$ is the planetary rotation rate, $b(t)=\sin \theta_{s}(t) \sin \theta$ and $c(t)=\cos$ $\theta_{s}(\mathrm{t}) \cos \theta$ with $\theta_{s}(\mathrm{t})$ being the (seasonally varying) latitude of the subsolar point. Thus, b, c, s, $\mathrm{q}$, and $\tau$ are all slowly varying on a time scale of one day. This suggests that the secular variations can be separated from the diurnal fluctuations by assuming

$$
T(t)-T_{e}=A_{0}(t)+\sum_{n}\left[A_{n}(t) \cos n \Omega t+B_{n}(t) \sin n \Omega t\right]
$$

and

$$
\exp [-\tau(z, t) / \mu(t)]=a_{0}(t)+\sum_{n} a_{n}(t) \cos n \Omega t
$$

where the coefficients in the expansions are slowly varying on a time scale of $\Omega^{-1}$. Then

$$
\left.\begin{array}{l}
a_{0}(t)=\frac{1}{\pi} \int_{0}^{\phi_{1}} \exp \left[-\frac{\tau(z, t)}{b(t) \cos \phi+c(t)}\right] d \phi \\
a_{n}(t)=\frac{2}{\pi} \int_{0}^{\phi_{1}} \exp \left[-\frac{\tau(z, t)}{b(t) \cos \phi+c(t)}\right] \cos n \phi d \phi
\end{array}\right\}
$$

where $\phi=\Omega \mathrm{t}$ and $\phi_{1}=\cos ^{-1}(-\mathrm{c} / \mathrm{b})$. The coefficients employed in the subsequent calculations were evaluated from (10) using numerical integration. 
Substituting (8) into (1) and using (4), (5) and (9), one obtains

$$
\begin{aligned}
& \frac{d \dot{A}_{0}}{d t}+\frac{\dot{A}_{0}}{t_{r}}=G(z, t) a_{0}(t), \\
& \frac{d A_{n}}{d t}+\frac{A_{n}}{t_{r}}+n \Omega B_{n}=G(z, t) a_{n}(t), \\
& \frac{d B_{n}}{d t}+\frac{B_{n}}{t_{r}}-n \Omega A_{n}=0 .
\end{aligned}
$$

The assumption that the coefficients change slowly on a time scale of $\Omega^{-1}$, along with the assumption that $t_{r}$ is of the order of one day or less allows the first terms on the left hand sides of each of equations (11) to be neglected. The coefficients can then be obtained and the solution to (1) written in the form

$$
T(z, t)=\bar{T}(z, t)+\sum_{n} \Delta T_{n}(t) \cos \left(n \Omega t-\delta_{n}\right)
$$

where

$$
\left.\begin{array}{l}
\bar{T}(z, t)=T_{e}(z)+t_{r} G(z, t) a_{0}(t) \\
\Delta T_{n}(z, t)=t_{r} G(z, t) a_{n}(t) / \sqrt{1+n^{2} \Omega^{2} t_{r}^{2}}
\end{array}\right\}
$$

The equilibrium temperature $T_{\mathrm{e}}$ for each level is taken to be the apparent asymptotic value approached by the temperature data in Figuree 1 and 2. The 
remaining parameters of the model are obtained by fitting the mean temperature $\mathrm{T}$ (t) and the diurnal amplitude $\Delta \mathrm{T}_{1}$ (t) to the observed data. A good fit is found by assuming

$$
\tau(z, t)=\tau(z, 0) e^{-t / t} c
$$

The resulting $\overline{\mathrm{T}}(\mathrm{t})$ (solid line) and $\overline{\mathrm{T}}(\mathrm{t}) \pm \Delta \mathrm{T}_{1}(\mathrm{t})$ (dashed lines) are shown in Figures 1 and 2 for $t_{c}=60$ days for both levels, and $t_{r}=5 \times 10^{4} \mathrm{sec}$. It is noteworthy that good fit is obtained at both levels with the same characteristic time $t_{c}$.

\section{Dust Dissipation}

Having estimated the temporal behavior of the extinction optical depth for solar radiation, it is of interest to consider the implications which can be drawn concerning the dust dissipation process. If it is assumed that during the dissipation phase no new dust particles are injected into the atmosphere and all particles reaching the ground remain there, then the dissipation is controlled by gravitational settling and vertical mixing. Since the gravitational settling rate is dependent on dust particle size, and the vertical mixing is generally parameterized in terms of an effective diffusivity, it should be possible in principle to obtain information on these parameters. To do this, it is necessary to define a model describing the dust dissipation.

It is assumed that the continuity equation governing the behavior of the suspended dust can be written

$$
\frac{\partial \mathrm{q}}{\partial \mathrm{t}}+\frac{1}{\rho} \frac{\partial g}{\partial z}=0
$$


where the vertical particle flux $F$ is

$$
g=w^{*} \rho \mathbf{q}-\mathbf{K} \rho \frac{\partial \mathbf{q}}{\partial z}
$$

The first term in (16) represents that portion of the total flux due to gravitational settling, and the second term is the contribution due to vertical mixing. The terminal velocity of the dust particles is denoted by $w^{*}$. All processes contributing to the vertical mixing of the dust are parameterized in terms of an effective vertical diffusivity $K$.

The terminal velocity required in (16) is assumed to be given by the StokesCunningham relation for spherical particles (Cunningham, 1910; Green and Lane, 1957; Ryan, 1964)

$$
w^{*}=-\frac{\rho_{\mathrm{p}} \mathrm{g} \mathrm{d}^{2}}{18 \eta}\left(1+2 \frac{\lambda}{\mathrm{d}}\right)
$$

where $\rho_{p}$ is dust particle density, $g$ is the Martian gravitational acceleration, $\mathrm{d}$ is particle diameter, $\eta$ is molecular viscosity, and $\lambda$ is molecular mean free path. The pressure dependence of $\lambda$ is approximated by

$$
\lambda(p) \cong \lambda\left(p_{r}\right) \frac{p_{r}}{p}
$$

where $p_{r}$ is an arbitrary reference pressure. In subsequent calculations, the following numerical values are used: $\rho_{\mathrm{p}}=3 \mathrm{gm} \mathrm{cm}^{-3}, \mathrm{~g}=370 \mathrm{~cm} \mathrm{sec}{ }^{-2}$, $\eta=1.5 \times 10^{-4} \mathrm{gm} \mathrm{sec} \mathrm{sm}^{-1}$, and $\lambda(25 \mathrm{mb})=2.2 \times 10^{-4} \mathrm{~cm}$. For the range of pressure and particle sizes considered here $2 \lambda / \mathrm{d} \gg>1$ so to good approximation $\mathrm{w}^{*}$ can be taken as proportional to $\mathrm{p}^{-1} \mathrm{~d}$. 
It is convenient to define a height-dependent characteristic settling time

$$
t_{s}(z)=\frac{H}{\left|w^{*}(z)\right|}
$$

where $\mathrm{H}$ is the pressure scale height. In the small particle limit where $\mathrm{w}^{*} \propto \mathrm{p}^{-1}$, $t_{s}(z)$ is the time required for the particle to fall from infinity to level $z$. In the large particle limit, $w^{*}$ is independent of $z$, and $t_{s}$ is the time required for the particle to fall through one scale height. Values of $t_{s}$ for the $0.3,2$, and $5 \mathrm{mb}$ levels are plotted as functions of particle diameter in Figure 3. In this calculation and in all subsequent calculations, it is assumed that $H=10 \mathrm{~km}$.

Consider first the conditions which existed during the storm prior to the onset of dissipation. In this case, it seems reasonable to assume a steady state (i.e., $\partial \mathrm{q} / \partial \mathrm{t}=0$ ), and therefore $\mathrm{g}=\mathrm{constant}$. If it is assumed that dust was injected into the atmosphere as rapidly as it was removed at the surface, then the net flux at the surface must vanish and $\mathcal{F}=0$ everywhere giving

$$
w^{*} q-K \frac{\partial q}{\partial z}=0
$$

Before proceeding further, it is necessary to make an assumption concerning the height dependence of $K$. At this point it will be assumed that $K$ is independent of height, and the effects of this assumption on the final results will be examined later. With the particle terminal velocity approximated by

$$
w^{*} \cong-w_{0}^{*} e^{z / H}
$$


where $-w_{0}^{*}$ is the value of $w^{*}$ at $z=0$, the solution of $(20)$ is

$$
q(z)=q_{0} \exp \left\{\nu\left[1-\exp \left(\frac{z}{H}\right)\right]\right\}
$$

where $q_{0}$ is the value of $q$ at $z=0$, and

$$
\nu=\frac{H w_{0}^{*}}{\mathrm{~K}}
$$

If a characteristic diffusion time is defined at $t_{D}=H^{2} / K$, then $\nu=t_{D} / t_{s}(0)$ where from (19) $t_{s}(0)$ is the characteristic settling time at $z=0$.

Examples of steady-state dust distributions calculated from (22) for selected values of $\nu$ are given in Figure 4. As the mixing is increased, $t_{\mathfrak{D}}$ and hence $\nu$ become smaller, and the dust extends to greater heights. In the limit of infinitely rapid mixing, $\nu \rightarrow 0$ and the specific concentration of the dust becomes independent of height.

It is convenient to define an effective "top" of the dust layer $Z_{t}$ at the point of inflection in the distribution (22) which can be written $Z_{t}=-H 1 n \nu$. This is the level at which the settling time $t_{s}(z)$ becomes equal to the diffusion time $t_{D}$. To maintain a dust distribution up to a given level $\mathrm{Z}_{t}$, the value of $\mathrm{K}$ required is dependent on the particle diameter. This dependence, calculated using (23) along with the definition of $z_{t}$, is shown in Figure 5 for several values of $Z_{t}$. Television pictures of the limb of the planet during the storm indicate dust up to at least $50 \mathrm{~km}$ (Leovy, et al., 1972). This suggests $\mathrm{Z}_{\mathrm{t}} \cong 50 \mathrm{~km}$ corresponding to $\nu=.007$ in $(22)$. 
Turning now to the problem of modeling the dissipative phase of the storm, time dependent solutions of (15) will be sought. It will be assumed that at time $t=0$, the mechanism for injecting dust into the atmosphere is switched off, and all particles reaching the surface remain there. The distribution for $\nu=.007$, which is shown as the broken curve in Figure 4, will be taken as the initial value of $q$ at $t=o$.

First consider the case when there is no mixing $(K=0)$, and (15) becomes

$$
\frac{\partial \mathrm{q}}{\partial \mathrm{t}}+\frac{1}{\rho} \frac{\partial}{\partial z}\left(w^{*} \rho \mathrm{q}\right)=0
$$

Using (21) and letting $\xi=p / p_{0} \cong e^{-z / H}$, the solution to (24) can be written

$$
q=f\left(\xi-\frac{w_{0}^{*}}{H} t\right)
$$

where $f(\xi)$ is an arbitrary function to be determined from the initial conditions. Using (22) and requiring that $q$ be non-negative,

$$
\mathrm{q}(\xi, \mathrm{t})= \begin{cases}\mathrm{q}_{0} \exp \left\{\nu\left[1-\exp \left(-\frac{1}{\xi-\mathrm{t} / \mathrm{t}_{\mathrm{s}}(0)}\right)\right]\right\} ; & \xi>\frac{\mathrm{t}}{\mathrm{t}_{\mathrm{s}}(0)} \\ 0 & ; \xi \leq \frac{\mathrm{t}}{\mathrm{t}_{\mathrm{s}}(0)}\end{cases}
$$

The optical depth, calculated from $(6)$, is 


$$
r(\xi \circ t)= \begin{cases}\sigma \mathrm{q}_{0} \rho_{0} \mathrm{e}^{\prime}\left[\xi-\frac{\mathrm{t}}{\mathrm{t}_{\mathrm{s}}(0)}\right] \mathrm{E}_{2}\left[\frac{\nu}{\xi-\mathrm{t} / \mathrm{t}_{\mathrm{s}}(0)}\right] ; \xi>\frac{\mathrm{t}}{\mathrm{t}_{\mathrm{s}}(0)} \\ 0 & ; \xi \leq \frac{\mathrm{t}}{\mathrm{t}_{\mathrm{s}}(0)}\end{cases}
$$

where $\mathrm{E}_{2}$ (x) is the exponential integral of second order defined as

$$
E_{2}(x)=\int_{1}^{\infty} \frac{e^{-x y}}{y^{2}} d y
$$

Figure 6 shows the optical depths at the 0.3 and $2 \mathrm{mb}$ levels as functions of time calculated from (27) with $\nu=.007$. The optical depth decreases much more rapidly at the upper level than at the lower level. Obviously this does not agree with the behavior deduced from the temperature data. Therefore, it must be concluded that vertical mixing played a significant role in the behavior of the dust during the dissipative phase of the storm as well as during the active phase.

It is now necessary to obtain solutions to (15) retaining both the gravitational settling and diffusive terms in the particle flux. Using (21) and $\mathrm{p} \cong \mathrm{p}_{0} \mathrm{e}^{-\mathrm{z} / \mathrm{H}}$, (15) can be written

$$
\frac{\partial q}{\partial t^{\prime}}=\frac{\partial^{2} q}{\partial z^{\prime 2}}+\left(\nu e^{z^{\prime}}-1\right)
$$

where the dimensionless indpendent variables are $t^{\prime}=t / t_{D}$ and $z^{\prime}=z / H$. Again the initial ralue is assumed to be given by (22) with $\nu=.007$. Note, however, that the value of $\nu$ appearing in (28) can in general have different value than that 
used in the initial condition since the value of $\mathrm{K}$ may have been different during the dissipative phase. In addition to the initial value, boundary conditions must also be specified. For $z \rightarrow \infty$ it is assumed that $q \rightarrow o$. Specification of the lower boundary condition has been discussed in connection with sedimentation problems in the terrestrial atmosphere by several authors (Johnstone, et al., 1949; Monin, 1959; Calder, 1961). Here it will be assumed that the diffusive portion of the flux must vanish at $z=0$. Since a constant value of $K$ has been assumed, this implies that $(\partial q / \partial z)_{z=0}=0$. Thus, dust particles are ultimately removed from an atmospheric column by pure gravitational settling out of the bottom of the column onto the planetary surface where they remain.

Solutions to (28) were obtained numerically for the initial value and boundary values discussed above. An implicit method of solution described by Richtmyer and Morton (1967) was employed. In practice $q$ was required to vanish at $z=100 \mathrm{~km}$. However, the exact choice of the level for which $q$ vanishes is not critical provided it is higher than the effective "top" of the initial distribution.

Calculations were made for a range of values of $\nu$. An example of the time evolution of $\mathrm{q}$ with $\nu=.01$ is shown in Figure 7. Optical depths at the 0.3 and $2 \mathrm{mb}$ levels obtained from $\mathrm{q}$ using (6) are shown in Figure 8 as functions of time. Examination of these curves indicates that the optical depth at the upper level decreases significantly more rapidly than that at the lower level for all values of $\nu$ greater than about .01. Thus, in order to obtain agreement with the optical depths inferred from the atmospheric temperature data it is necessary to choose $\nu \lesssim .01$

From Figure 8d, the time dependence of the optical depths is essentially exponential with an e-folding time of approximately $t / t_{s}(0)=1.2$. For $t=60$ 
days as inferred from the temperature data, $t_{s}(o)=50$ days. Assuming an atmospheric pressure at the surface of $5 \mathrm{mb}$, this settling time corresponds to a dust particle diameter of $2.3 \mu \mathrm{m}$ (Figure 3). From a knowledge of the settling time and the limit established on $\nu$, it is found from (23) that $\mathrm{K} \gtrsim 2 \times 10^{7} \mathrm{~cm}^{2}$, $\mathrm{sec}^{-1}$. With a knowledge of the particle diameter, it is also possible to estimate the value of $\mathrm{K}$ required during the active phase of the storm to maintain dust up to $\sim 50 \mathrm{~km}$. From Figure 5 with $\mathrm{d} \sim 2 \mu \mathrm{m}$, it is found that $\mathrm{K} \sim 10^{7} \mathrm{~cm}^{2} \mathrm{sec}^{-1}$ is needed. Thus, the model considered here requires similar values of $\mathrm{K}$ during both the active and dissipative phases of the storm.

The assumption of constant $\mathrm{K}$ has resulted in large values for this parameter, corresponding to strong mixing at all atmospheric levels up to at least $50 \mathrm{~km}$. To investigate the dependence of the results on the height variation of $\mathrm{K}$, calculations were made for two additional models. Results obtained with $K(z)=K_{0} \exp (z / 2 H)$ are shown in Figure 9 for $w_{0}^{*} H / K_{0}=.05$; larger values of this parameter result in a more rapid decay in $\tau$ at the upper level. From the figure, the e-folding time is $t / t_{s}(0) \cong 0.9$ which for $t=60$ days results in $d \cong 1.8 \mu \mathrm{m}$ and $\mathrm{K}_{0}=4 \times 10^{6}$ $\mathrm{cm}^{2} \mathrm{sec}^{-1}$. This particular form of $\mathrm{K}(\mathrm{z})$ is of interest since it is representative of mixing induced by vertically propagating gravity waves (Cf. Wofsy and McElroy, 1973). As an extreme case of height dependence, solutions were also obtained with $\mathrm{K}(\mathrm{z})=\mathrm{K}_{0} \mathrm{e}^{\mathrm{z} / \mathrm{H}}$ with the results shown in Figure 10 for $\mathrm{w}_{0}^{*} \mathrm{H} / \mathrm{K}_{0}=0.2$. Again requiring an e-folding time of 60 days, it is found that $K_{0}=9 \times 10^{5} \mathrm{~cm}^{2}$ $\sec ^{-1}$ and $\mathrm{d} \cong 1.4 \mu \mathrm{m}$.

The diffusivity profiles obtained with the three different models are summarized in Figure 11. Also shown is the profile required for the diffusion time to be equal to the gravitational settling time at all levels. It is not 
possible to derive a unique height dependence for $\mathrm{K}$ on the basis of the data analyzed here. However, the basic picture emerging from the calculations can be summarized as follows. The secular dependence of the optical depths as inferred from atmospheric temperature measurements can be explained in terms of models in which the dust is strongly mixed up to $\sim 50 \mathrm{~km}$. The residence time of the dust is determined primarily by the gravitational settling rate at the bottom of an atmospheric column. For this reason, the particle size inferred from the residence time does not depend strongly on the height profile of $\mathrm{K}$. To maintain a strongly mixed state within an atmospheric column, the characteristic diffusion time must be of the order of or less than the gravitational settling time at each level. The values of $\mathrm{K}$ shown represent the minimum amount of mixing required for each model and therefore represent lower limits. Between the surface and $50 \mathrm{~km}$, the mean values of $\mathrm{K}$ for the two height dependent models are of the same order as the value of $\mathrm{K}$ obtained with the height independent model.

The particle size estimate of $d \sim 2 \mu \mathrm{m}$ obtained here is comparable with most of the previous estimates which have been made using other techniques. Moroz and Ksanfomaliti (1972), using infrared photometry at 0.7 and $1.4 \mu \mathrm{m}$ from Mars 3, estimate the particle size to be about $1 \mu \mathrm{m}$ while Conrath, et al. (1973) find a mean particle radius between 1 and $10 \mu \mathrm{m}$ from the characteristics of the mineral dust features in the infrared spectra. Pang and Hord (1973) estimate the mean radius to be about $2 \mu \mathrm{m}$ based on ultra violet spectrometer data from Mariner 9. Leovy, et al. (1972) infer somewhat larger particles with mean radii in excess of $10 \mu \mathrm{m}$, based on television photometry from Mariner 9. 
Recently, Hartmann and Price (1974) have examined the time dependence of the contrast of surface features observed in the Mariner 9 television pictures, obtaining particle sizes $\lesssim 5 \mu \mathrm{m}$ in the upper atmosphere and $\lesssim 10 \mu \mathrm{m}$ in the lowest layers.

\section{Conclusions}

Atmospheric temperatures derived from the infrared spectroscopy investigation on Mariner 9 during the dissipation of the dust storm of 1971 reveal the manner in which the atmosphere relaxed from the perturbed thermal state which existed during the storm. It is found that the observed secular changes in mean temperatures and diurnal amplitudes can be well fit with a highly simplified radiative heating model. Within the framework of this model, it is possible to estimate the time dependence of the optical depth of the dust above the $0.3 \mathrm{mb}$ and $2 \mathrm{mb}$ levels. An approximately exponential behavior with the same characteristic time (60 days) is found for each level.

Since Stokes-Cunningham settling alone would result in clearing at a more rapid rate above the $0.3 \mathrm{mb}$ level than above the $2 \mathrm{mb}$ level, it is concluded that vertical mixing must have played a significant role during the dissipation phase. Applications of dust dissipation models which include both gravitational settling and vertical mixing, indicate that the mean diameter of the particles responsible for the heating was approximately $2 \mu \mathrm{m}$. It is not possible to uniquely establish the height dependence of the vertical diffusivity $\mathrm{K}$ from the data considered here. With the assumption of a height independent diffusivity, it is found that $\mathrm{K} Z 10^{7}$ $\mathrm{cm}^{2} \mathrm{sec}^{-1}$ is required to maintain sufficient vertical mixing to produce the observed behavior of the optical depths during the dissipation of the storm. 
A similar value of $\mathrm{K}$ would have been required during the active phase of the storm to maintain a steady state distribution of $2 \mu \mathrm{m}$ size particles up to $50 \mathrm{~km}$.

\section{ACKNOWLEDGEMENTS}

The author is indebted to J. A. Pirraglia and P. J. Gierasch for useful discussions of the subject matter of this paper. The Martian atmospheric temperatures were obtained in collaboration with V. G. Kunde, J. C. Pearl and R. A. Hanel, Principle Investigator of the Mariner 9 Infrared Spectroscopy Investigation, who also provided a critical review of the manuscript. H. W. Sagges provided the computer programming necessary for carrying out the numerical calculations.

\section{REFERENCES}

Calder, K. L. (1961). Atmospheric diffusion of particulate material considered as a boundary value problem. Journal of Meteorology 18, 413-416.

Conrath, B.J., and Revah, I. (1972). A review of nonstatistical techniques for the estimation of vertical atmospheric structure from remote infrared measurements. In "Proceedings of a Workshop on the Mathematics of Profile Inversion" (L. Colin, Ed.), NASA TMX-62150.

Conrath, B., Curran, R., Hanel, R., Kunde, V., Maguire, W., Pearl, J., Pirraglia, J., and Welker, J. (1973). Atmospheric and surface properties of Mars obtained by infrared spectroscopy on Mariner 9 . J. Geophys. Res. 78 , $4267-4278$.

Cunningham, E. (1910). On the velocity of steady fall of spherical particles through fluid medium. Proc. Roy. Soc. London (Series A) 83, 357-365. 
Gierasch, P. and Goody, R. (1968). A study of the thermal and dynamical structure of the Martian lower atmosphere. Planet. Space Sci. 16, 615-646.

Gierasch, P., and Goody, R. (1972). The effect of the dust on the temperature of the Martian atmosphere. J. Atmos. Sci. 29, 400-402.

Green, H. L., and Lane, W. R. (1957). "Particulate Clouds", E.\& F. N. Spon Ltd., London.

Hanel, R. A., Conrath, B. J., Hovis, W. A., Kunde, V. G., Lowman, P. D., Pearl, J. C., Prabhakara, C., and Schalchman, B. (1972a). Infrared spectroscopy experiment on the Mariner 9 mission: Preliminary results. Science 175, 305-308。

Hanel, R., Conrath, B., Hovis, w., Kunde, V., Lowman, P., Maguire, W., Pearl, J., Pirraglia, J., Prabhakara, C., and Schlachman, B. (1972b), Investigation of the Martian environment by infrared spectroscopy on Mạiner 9。 Icarus 17, 423-442.

Hartmann, W. K., and Price, M.J. (1974). Mars: Clearing of the 1971 dust storm. Icarus 21, 28-34.

Johnstone, H, F., Winsche, W. E., and Smith, L, W., (1949). The dispersion and deposition of aerosols. Chem: Rev. 44, 353-371.

Leovy, C. B., Briggs, G. A., Young, A. T., Smith, B. A., Pollack, J. B., Shịpley, E. N., and Widey, R. L. (1972). The Martian atmosphere: Mariner 9 television experiment progress report. Icarus 17, 373-393.

Leovy, C. B., Zureck, R. W., and Pollack, J. B. (1973)。 Mechanisms for Mars dust storms. J. Atmos. Sci. 30, 749-762. 
Monin, A. S. (1959). On the boundary condition on the earth surface for diffusing pollution, in "Advances in Geophysics" (Vol. 6), pp. 435-436. Academic Press, New York.

Moroz, V. I., and Ksanfomaliti, L. V. (1972). Preliminary results of astrophysical observations of Mars from Mars 3. Icarus $17,408-422$ 。

Pang, K. and Hord, C. W. (1973). Mariner 9 Ultraviolet spectrometer experiment: 1971 Mars' dust storm. Icarus 18, 481-488.

Pirraglia, J. A., and Conrath, B. J: (1974). Martian tidal pressure and wind fields obtained from the Mariner 9 infrared spectroscopy experiment. J. Atmos. Sci. 31, 318-329.

Richtmyer, R. D., and Morton, K. W. (1967). "Difference Methods for InitialValue Problems" (2nd edition), pp. 198-201. Interscience, New York.

Ryan, J. A. (1964). Notes on the Martian yellow clouds. J. Geophys. Res. 69 , $3759-3770$.

Wofsy, S. C., and McElroy, M. B. (1973). On vertical mixing in the upper stratosphere and lower mesosphere. J. Geophys. Res. 78, 2619-2624. 


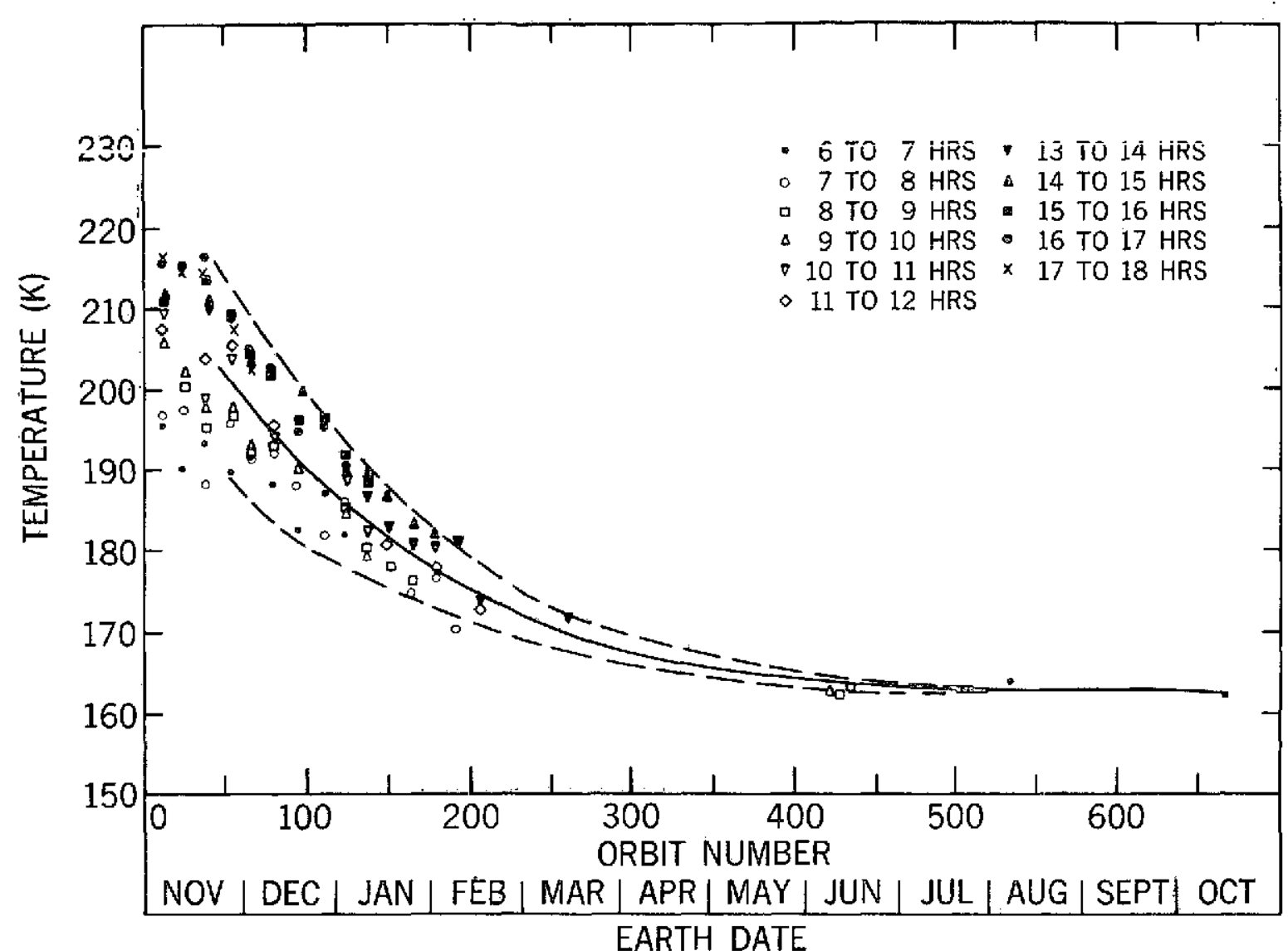

Figure 1. Secular variation of atmospheric temperatures at the $0.3 \mathrm{mb}$ level for the latitude interval $-20^{\circ}$ to $-30^{\circ}$. The abscissa is Mariner 9 orbit number with the corresponding earth date also shown. Data points prior to orbit 210 represent averages over approximately 6 days while data points after orbit 210 represent individual measurements. The data have been orgainized by Martian local time as indicated in the key to the various symbols. The solid line represents the mean temperature and the broken lines the limit of the diurnal excursion calculated using a heating model described in the,text. 


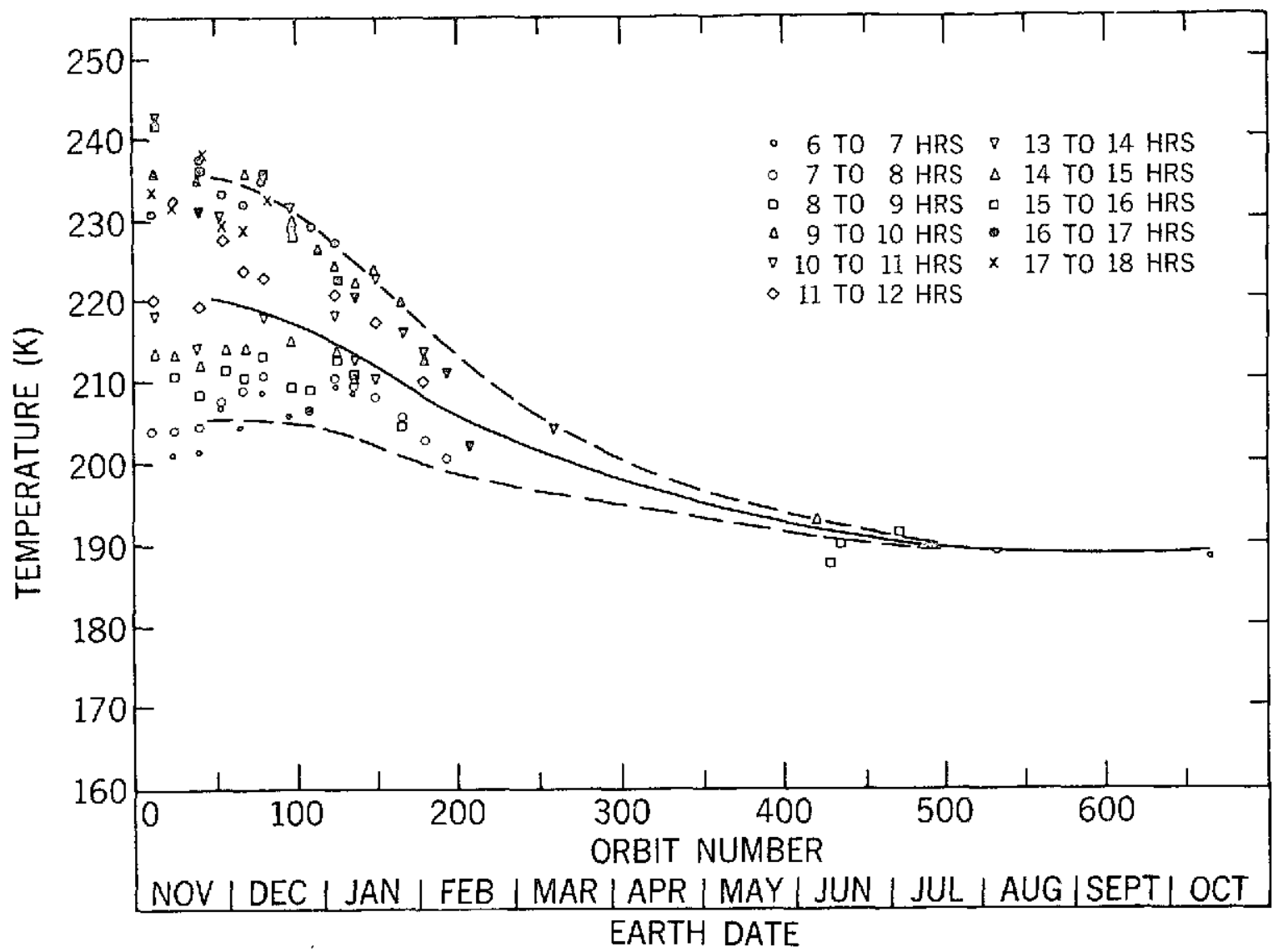

Figure 2. Secular variation of atmospheric temperatures at the $2 \mathrm{mb}$ level for the latitude interval $-20^{\circ}$ to $-30^{\circ}$. The abscissa is Mariner 9 orbit number with the corresponding earth date also shown. Data points prior to orbit 210 represent averages over approximately 6 days while data points after orbit 210 represent individual measurements. The data have been organized by Martian local time as indicated in the key to the various symbols. The solid line represents the mean temperature and the broken lines the limit of the diurnal excursion calculated using a heating model described in the text. 


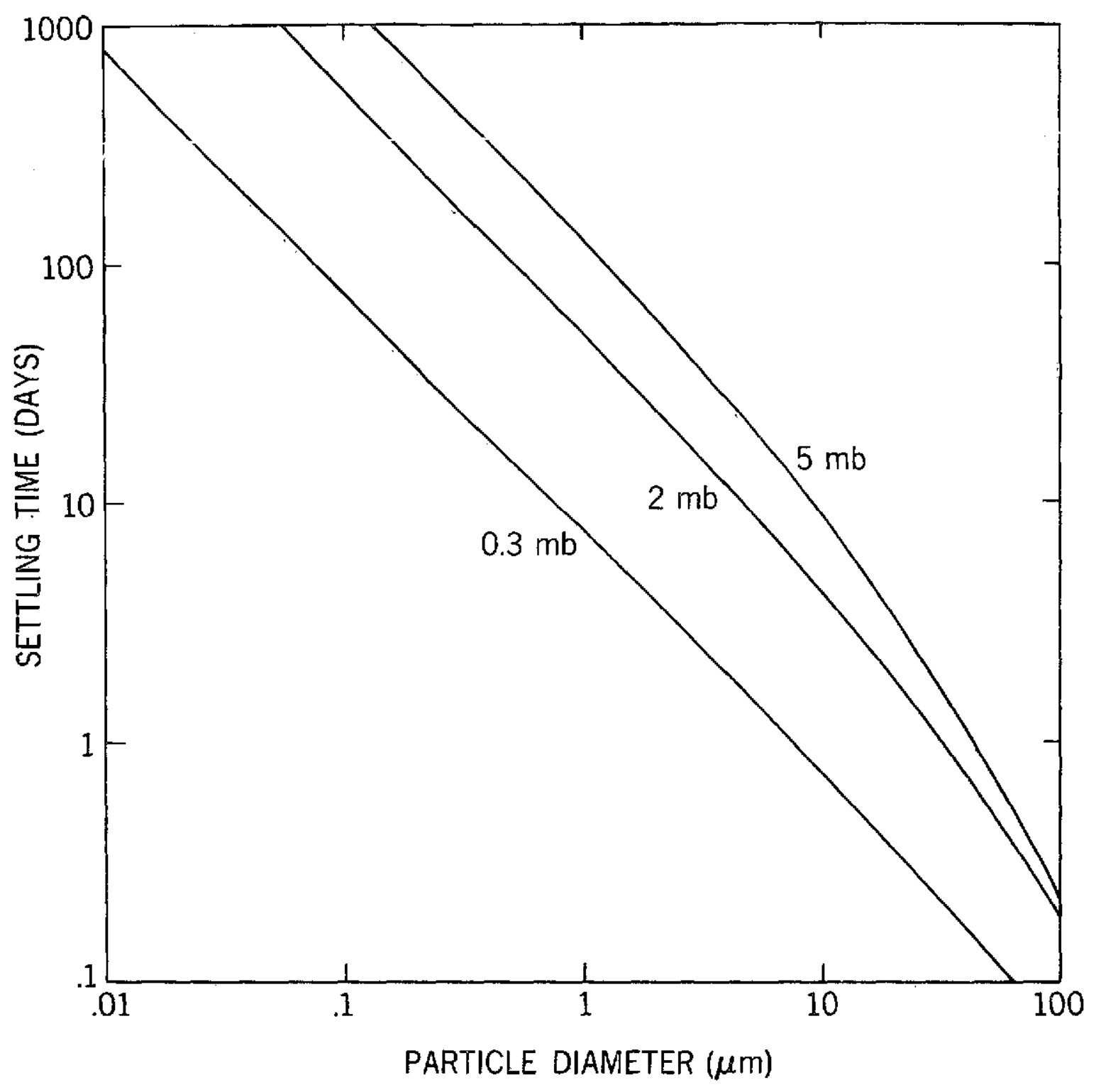

Figure 3. Gravitational settling times for dust particles versus particle diameter for three atmospheric pressure levels. The settling time is defined by equation (19) in the text. 


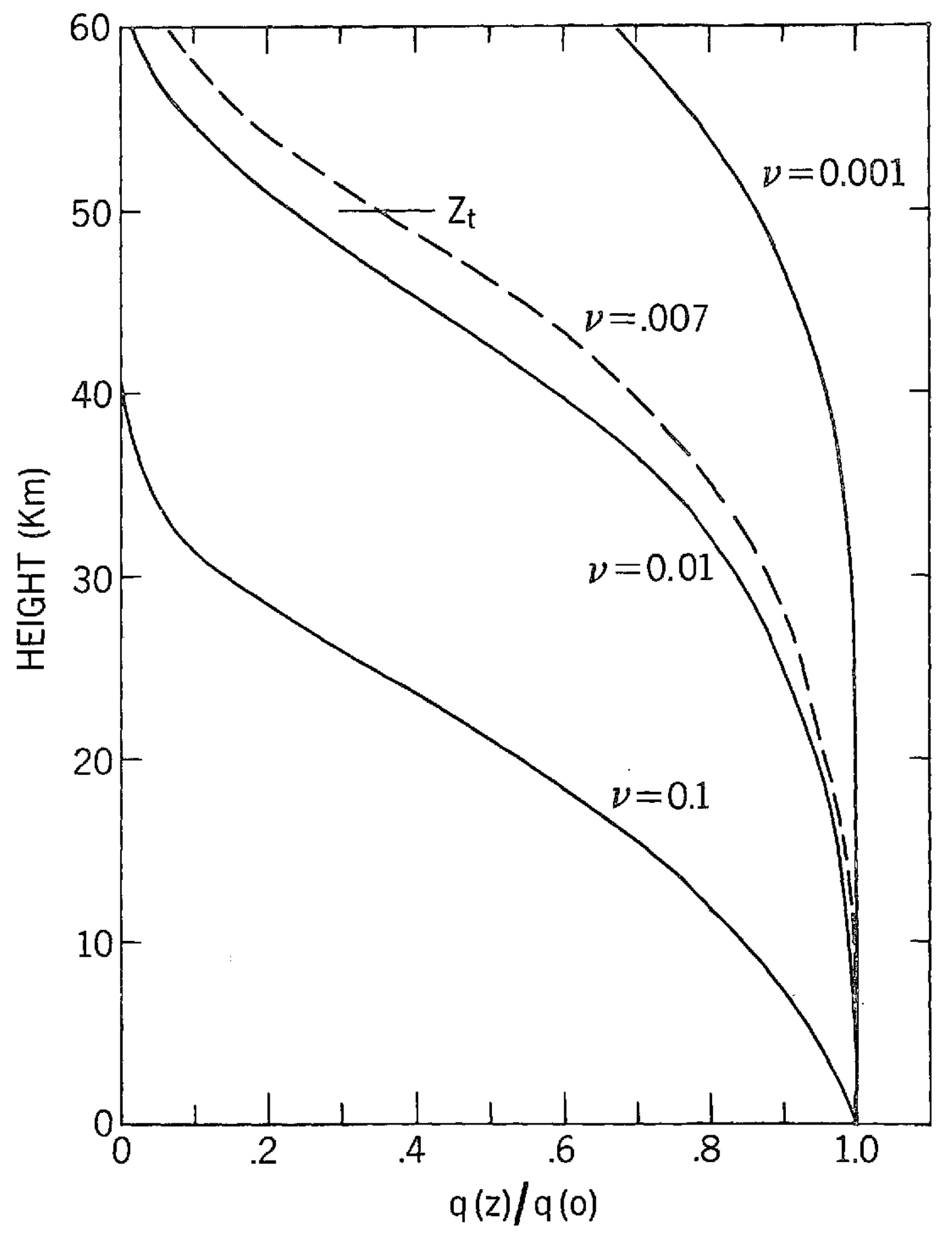

Figure 4. Vertical dust profiles obtained assuming steady state conditions and a constant vertical diffusivity $\mathrm{K}$. Curves are shown for selected values of the parameter $\nu$, the ratio of the characteristic time to the gravitational settling time at the planetary surface. The specific dust concentration $q$ is normalized to its value at the surface. 


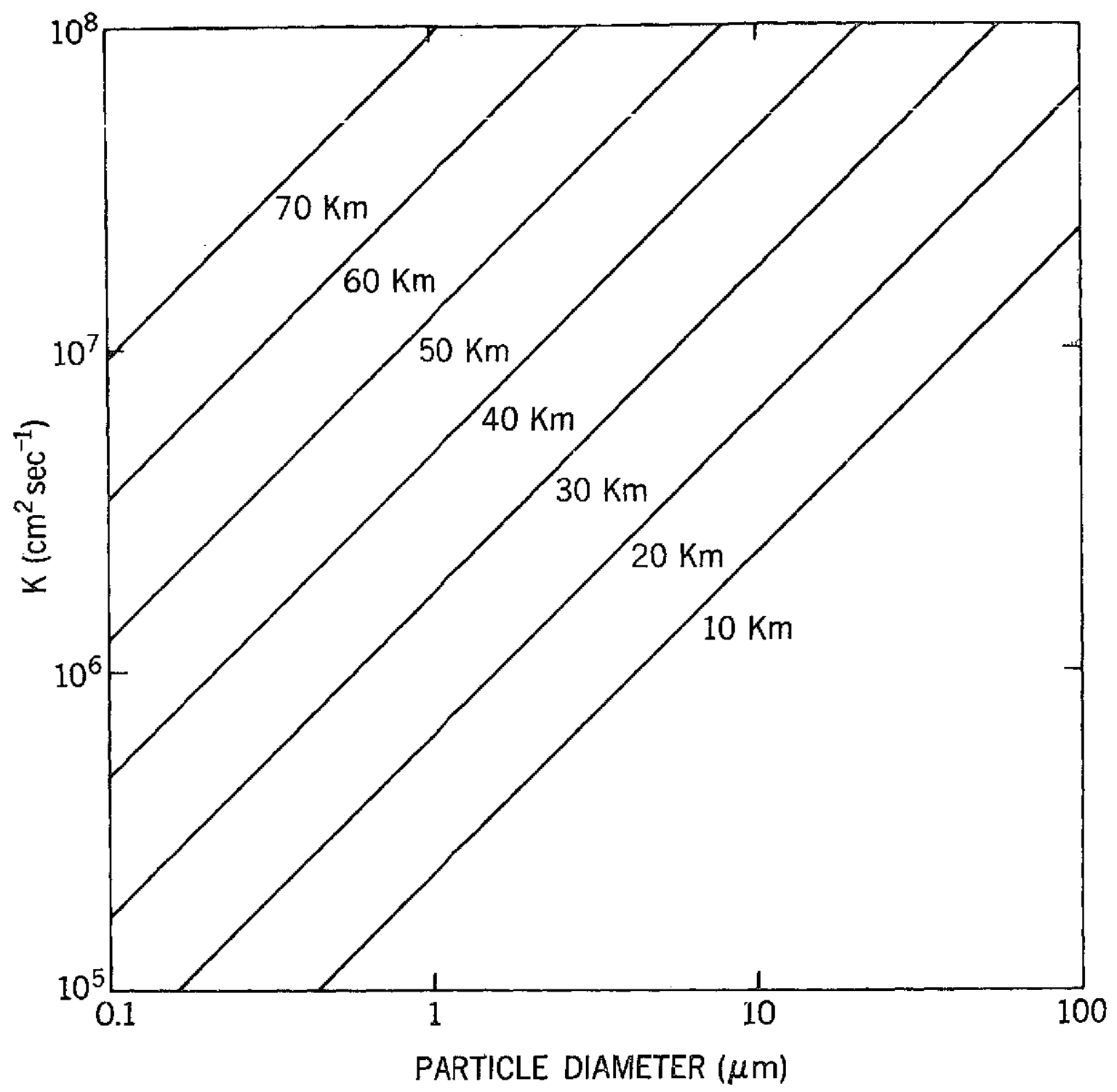

Figure 5. Vertical diffusivity $\mathrm{K}$ required to maintain dust particles well mixed to the height indicated on each curve. $\mathrm{K}$ was assumed to be independent of height for these calculations. 


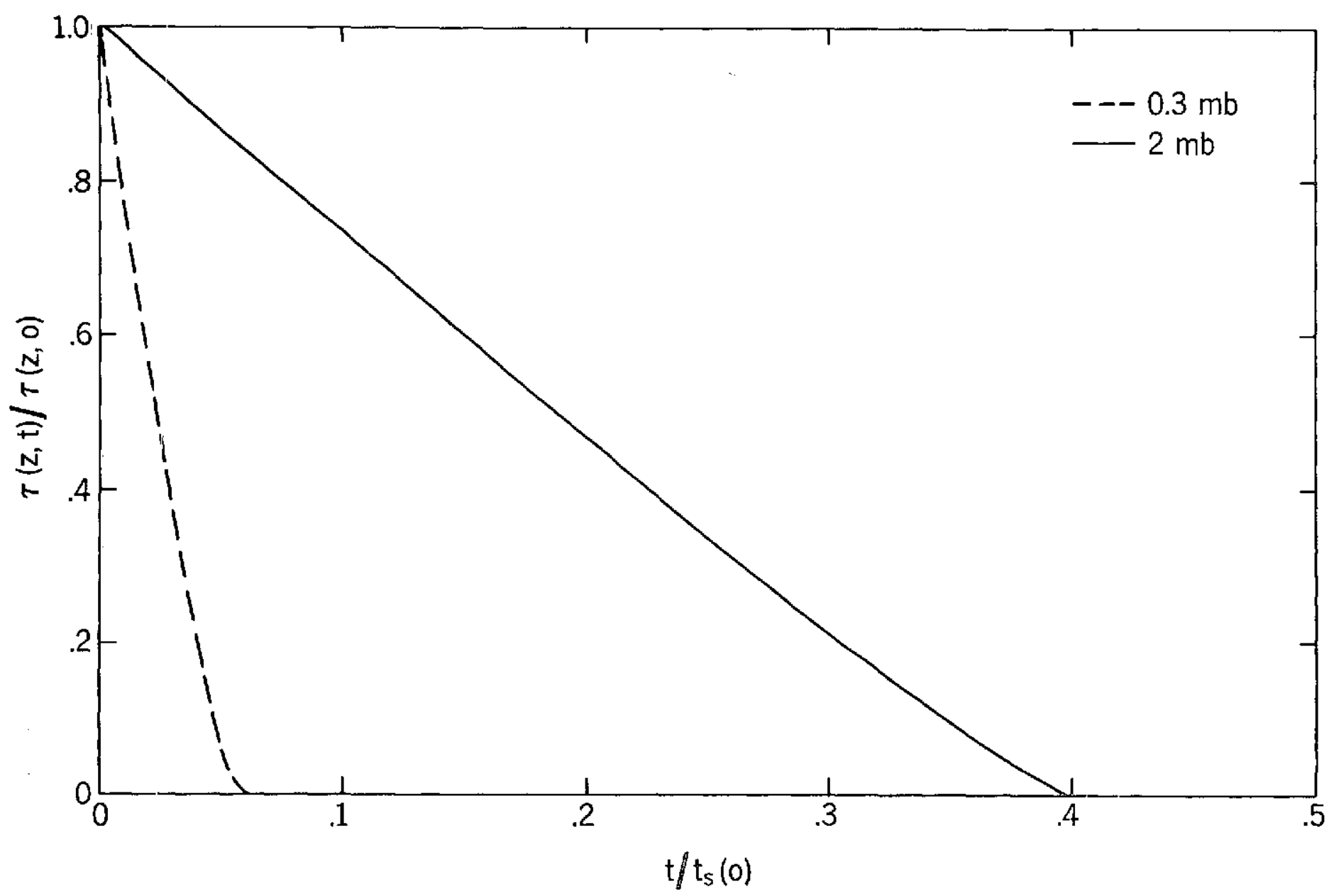

Figure 6. Calculated time dependence of optical depths at the 0.3 and $2 \mathrm{mb}$ levels assuming gravitational settling only. The optical depth at each level is normalized to its value at time $t=0$. The time scale is normalized to $t_{s}(0)$, the gravitational settling time at the surface. 


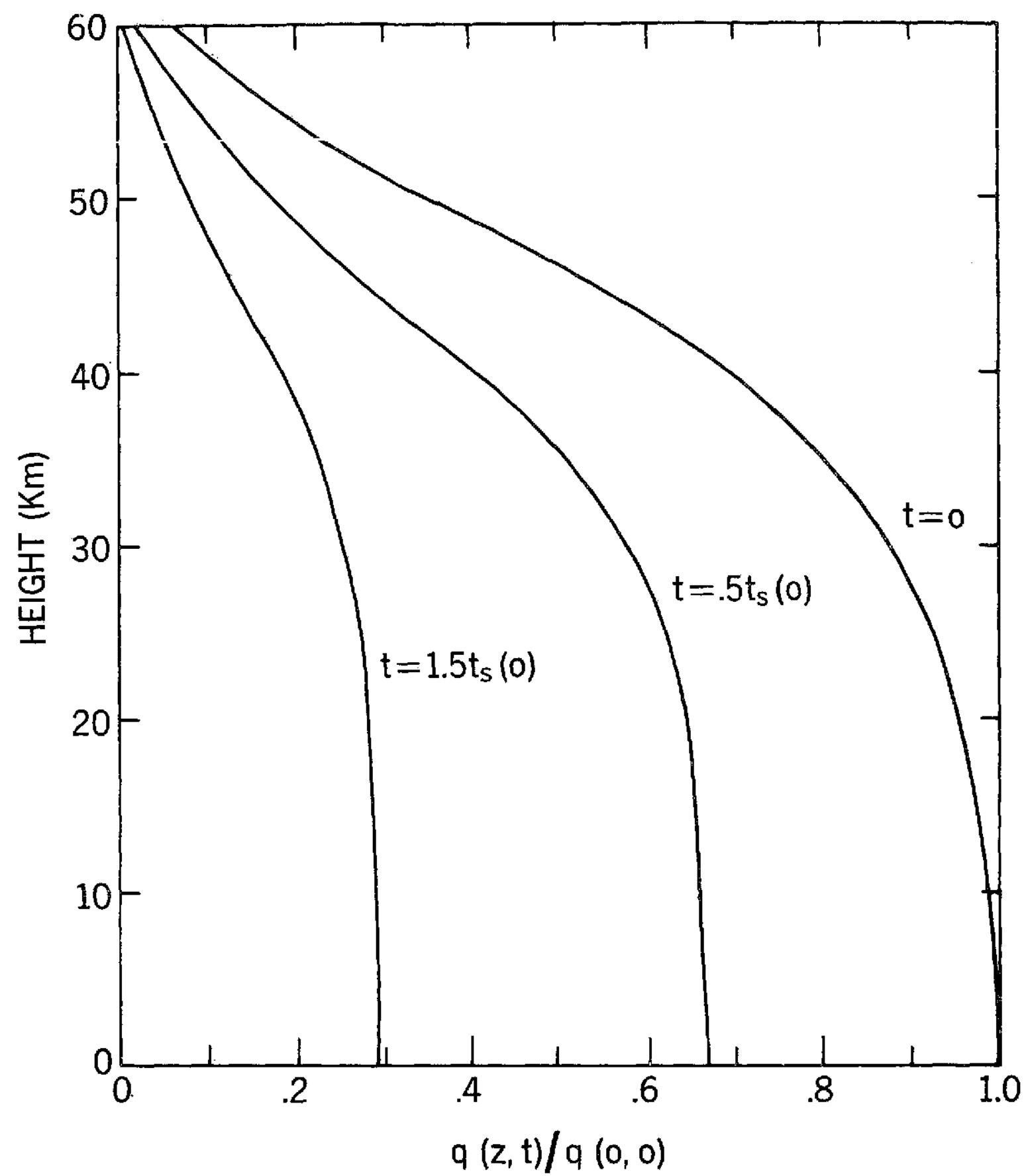

Figure 7. Example of the time evolution of the vertical dust profile obtained with $\nu=.01$ in the dissipation model discussed in the text. The specific dust concentration $q$ is normalized to its value at the surface at time $t=0$, and the times indicated for each curve are expressed in terms of the gravitational settling time at the surface. 


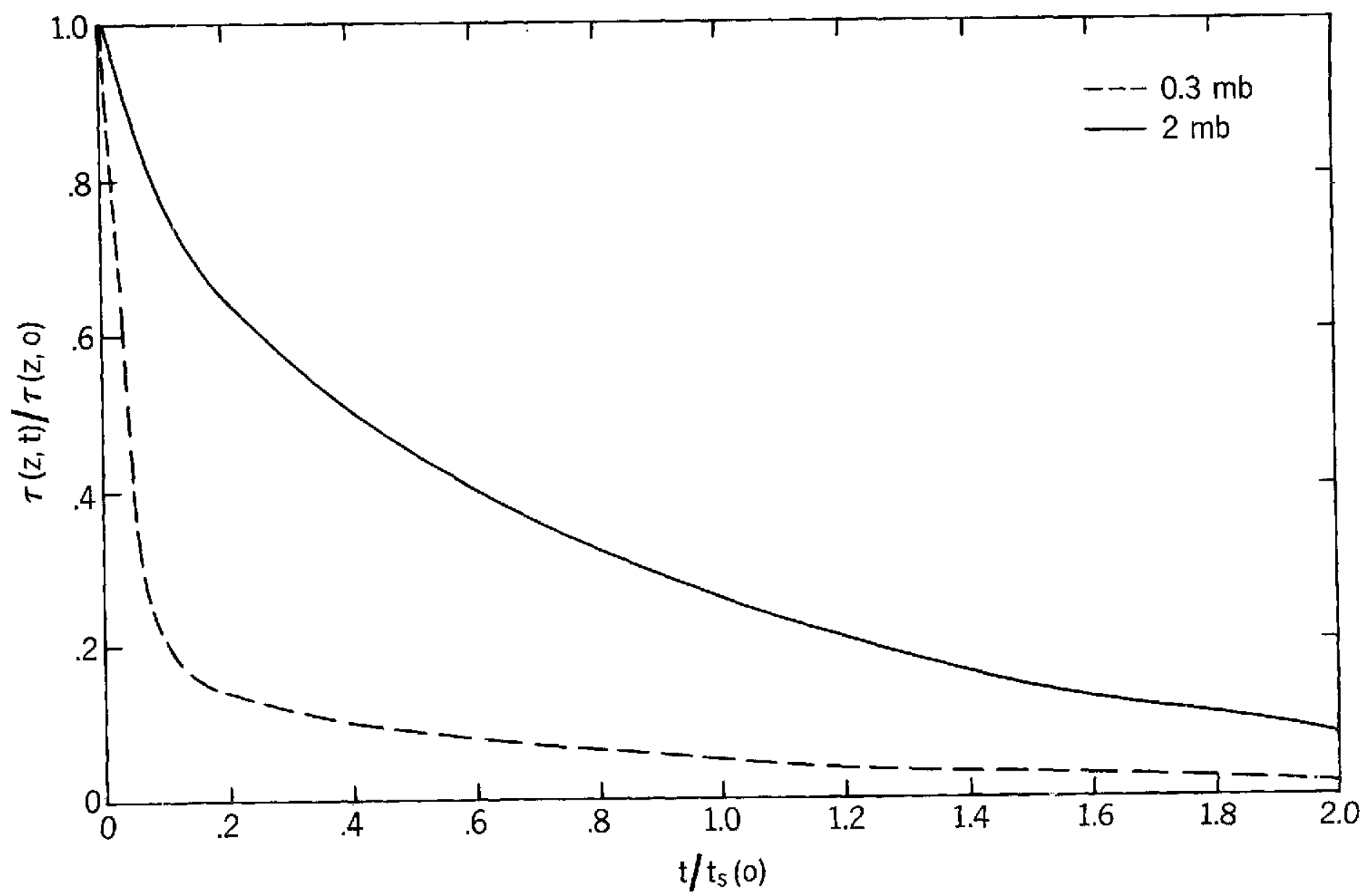

(a) $\nu=0.1$

Figure 8. Calculated time dependence of optical depths at the 0.3 and $2 \mathrm{mb}$ levels assuming both gravitational settling and vertical mixing. The optical depth at each level is normalized to the gravitational settling at the surface. Results are shown for selected values of $\nu$, the ratio of the characteristic diffusion time to the gravitational settling time at the surface: (a) $\nu=0.1$, (b) $\nu=0.05$; (c) $\nu=0.02$; (d) $\nu=0.01$. A height independent vertical diffusivity was assumed. 


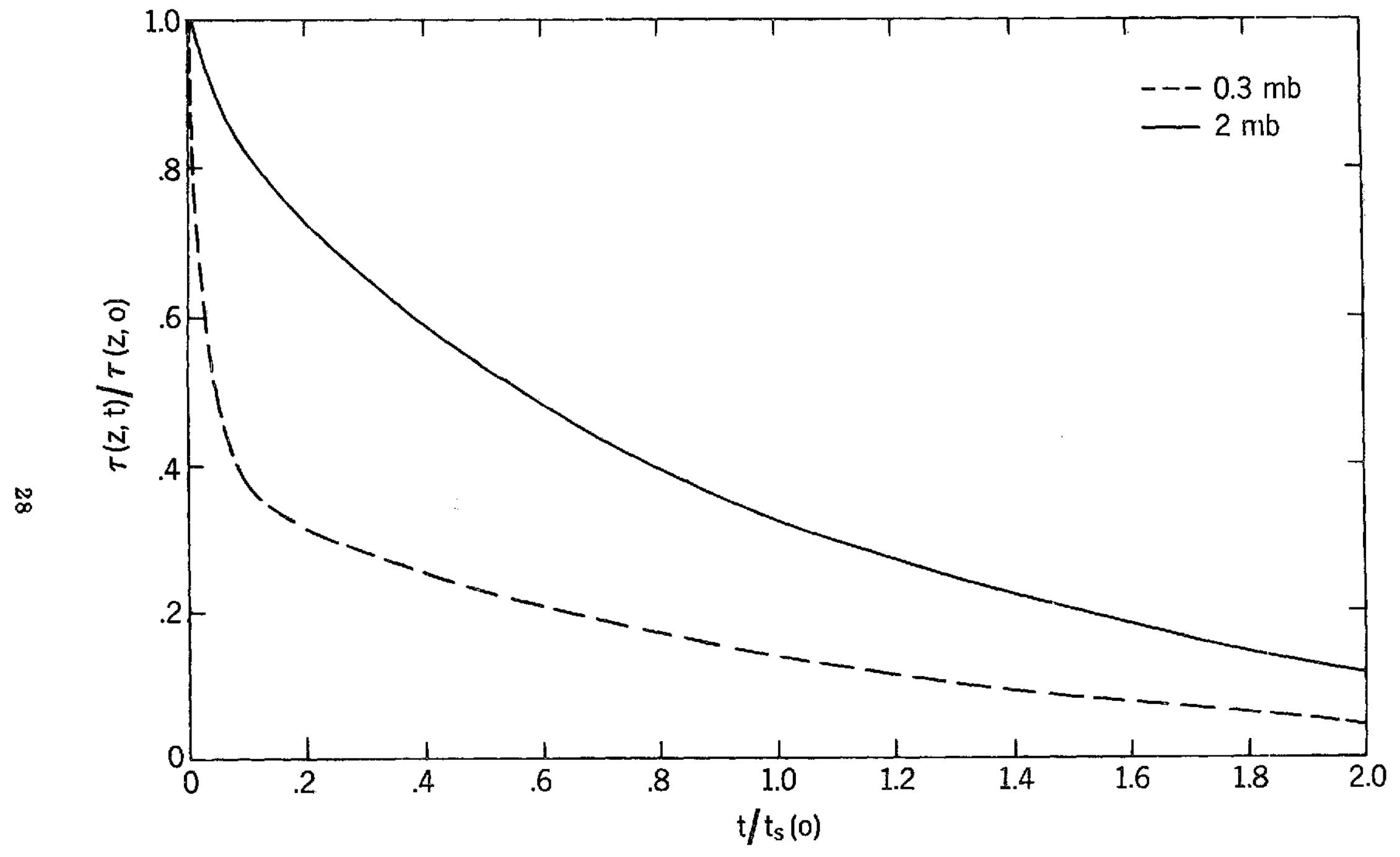

(b) $\nu=0.05$

Figure 8. (continued). 


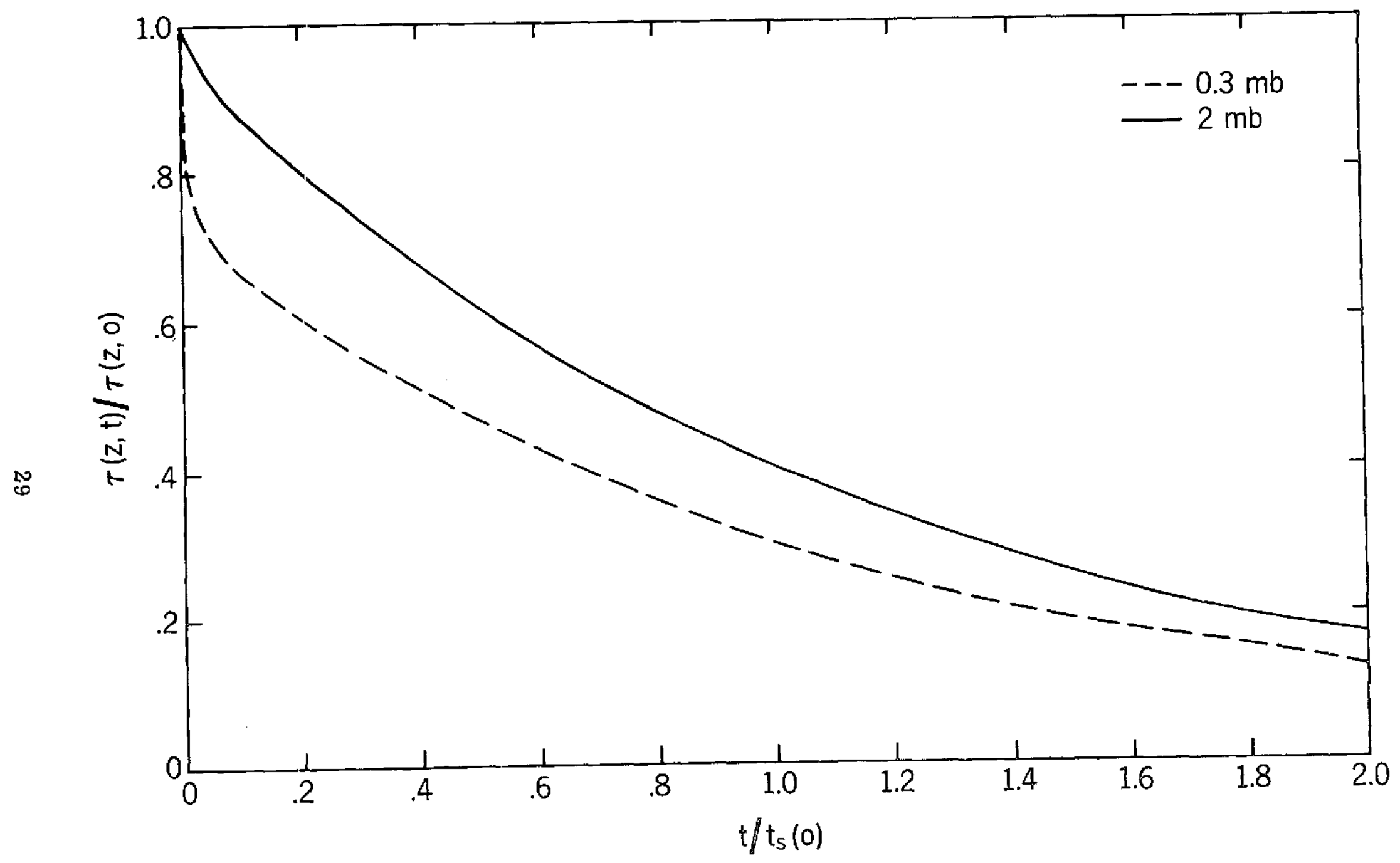

(c) $\nu=0.02$

Figure 8. (continued). 


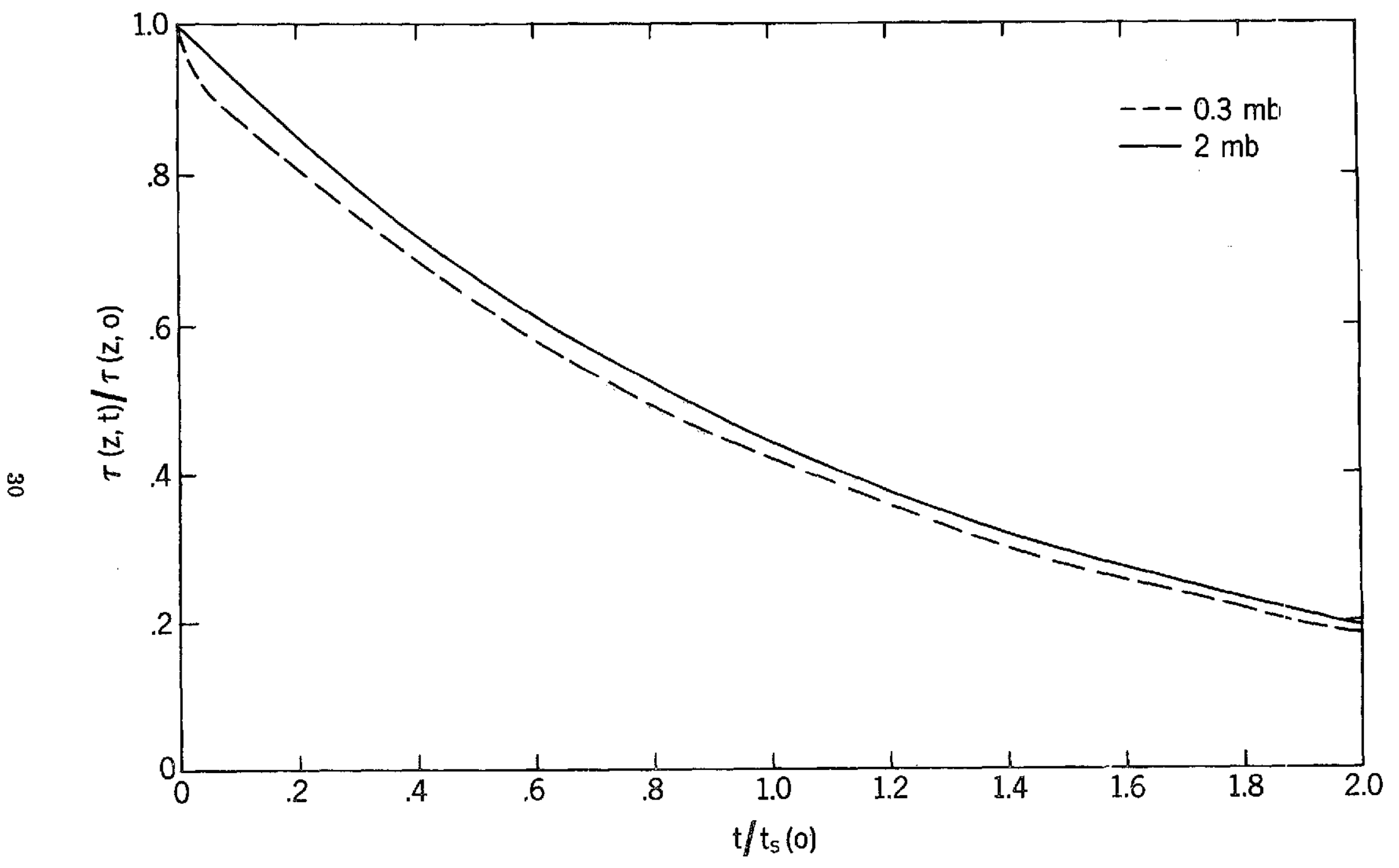

(d) $\nu=0.01$

Figure 8. (concluded). 


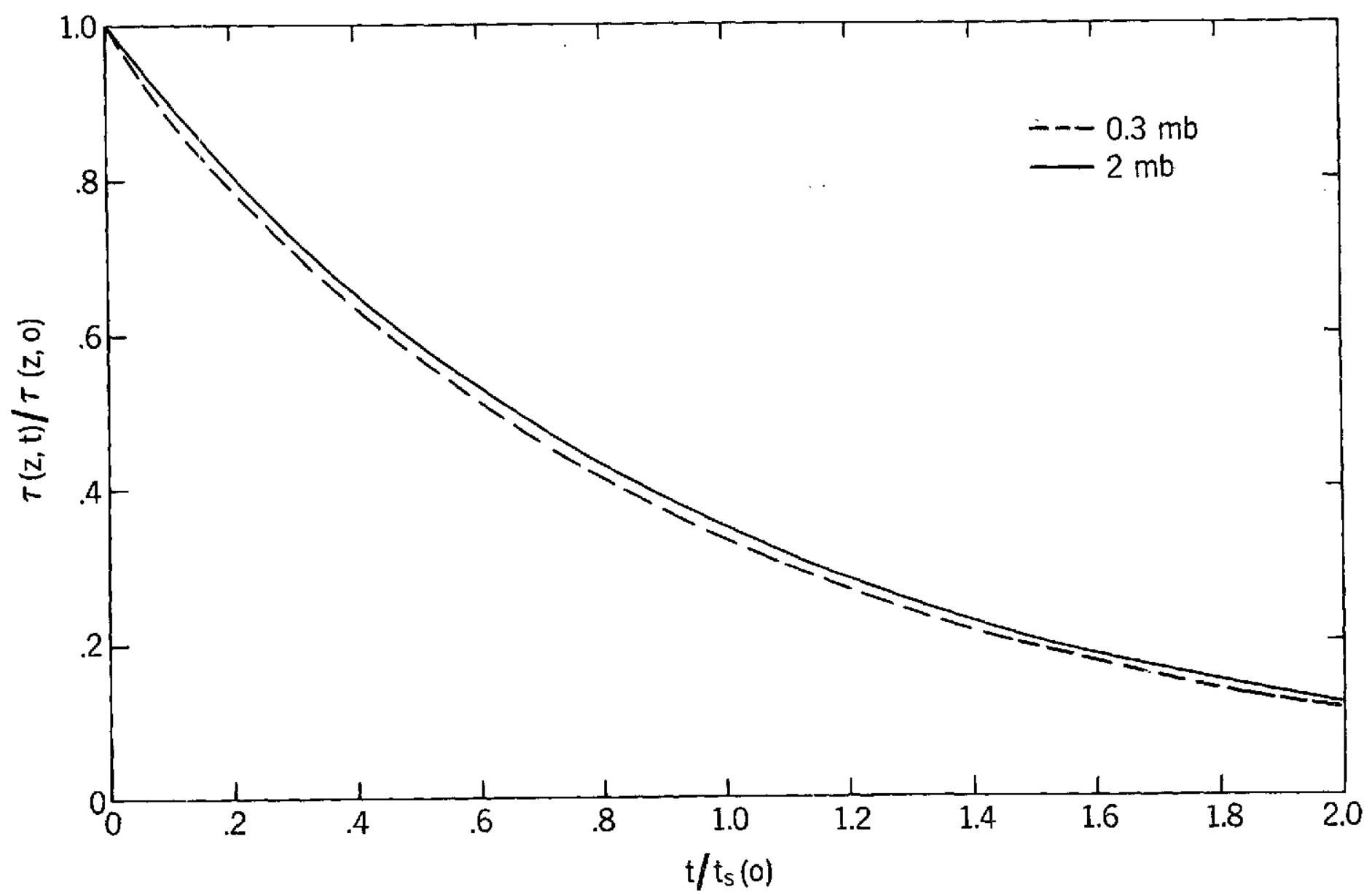

Figure 9. Calculated time dependence of optical depths at the 0.3 and $2 \mathrm{mb}$ levels assuming a vertical diffusivity $\mathrm{K}=\mathrm{K}_{0} \exp (\mathrm{z} / 2 \mathrm{H})$. In this example, the ratio of the characteristic diffusion time to gravitational settling time at the surface is 0.05 . 


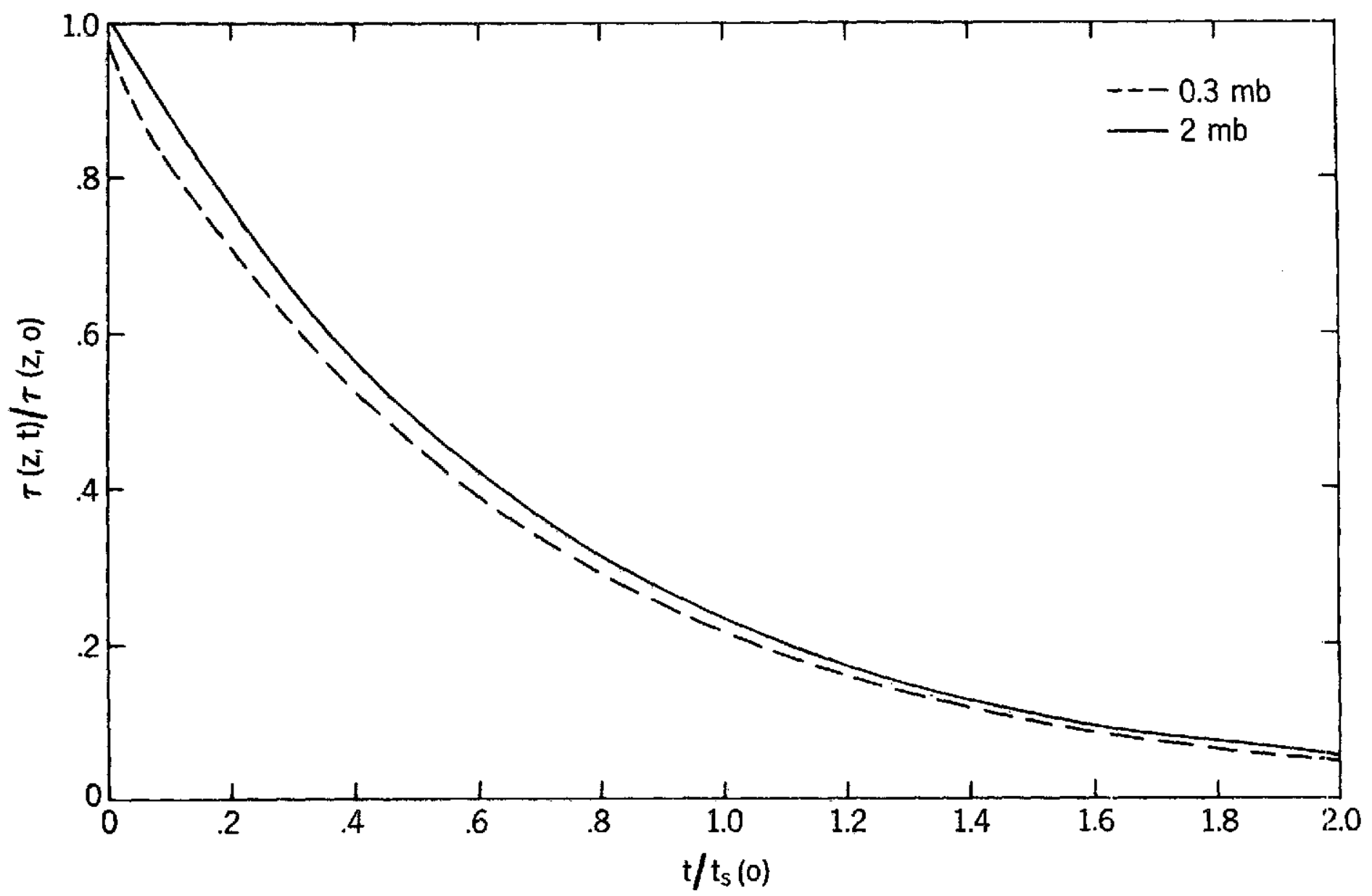

Figure 10. Calculated time dependence of optical depths at the 0.3 and $2 \mathrm{mb}$ levels assuming a vertical diffusivity $K=K_{0} \exp (\mathrm{z} / \mathrm{H})$. In this example the ratio of the characteristic diffusion time to the gravitational settling time at the surface is 0.2 . 


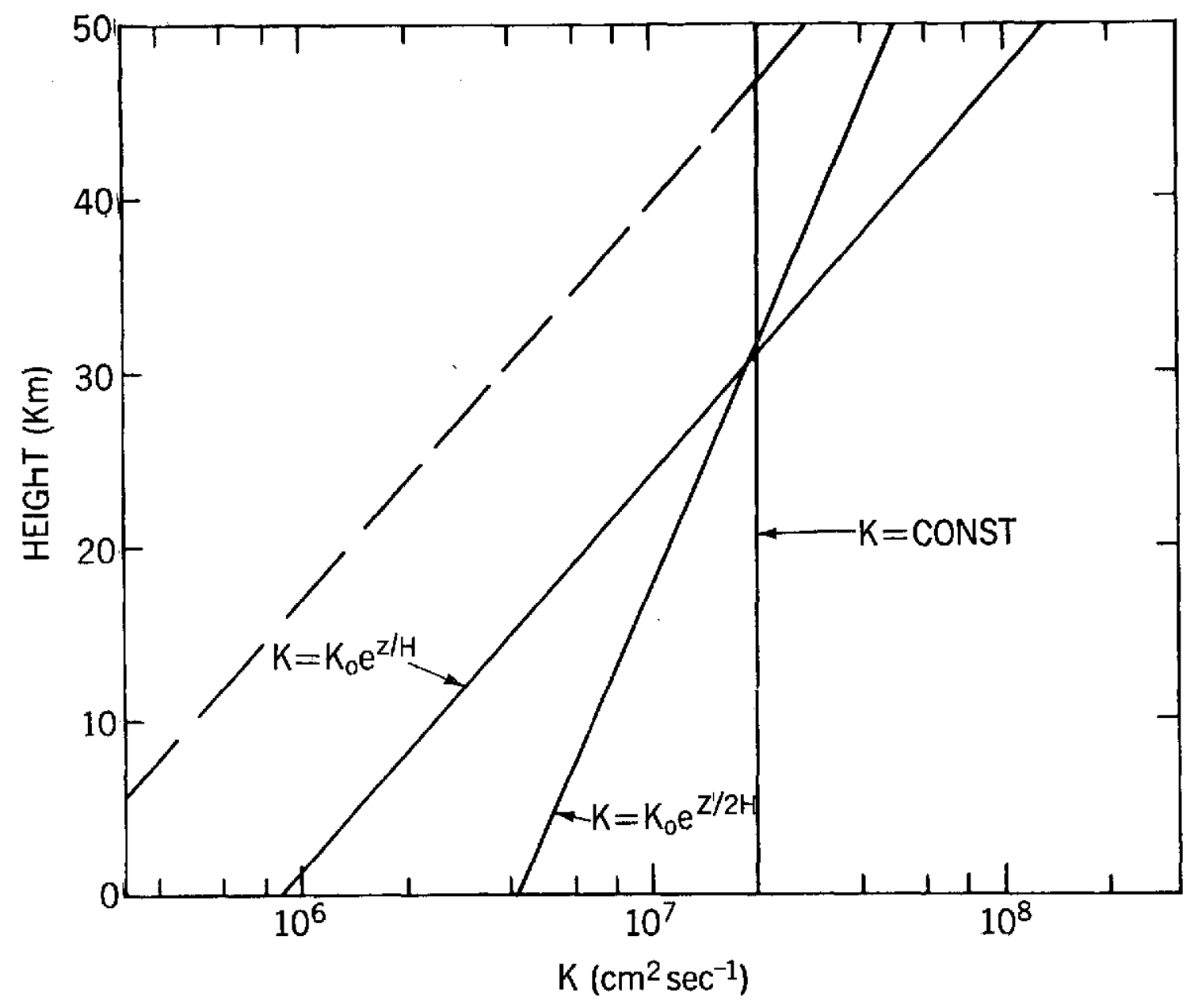

Figure 11. Comparison of the three models of $\mathrm{K}$ employed to obtain the results shown in Figures 8, 9, and 10. The broken line indicates the value of $\mathrm{K}$ required for the characteristic diffusion time to be equal to the gravitational settling time at each level for $2 \mu$ particles. 